\title{
Energy Expenditure of Standing Compared to Sitting While Conducting Office Tasks
}

\author{
Jill Burns, Cuisle Forde, and Sara Dockrell, Trinity College, Dublin, Ireland
}

Objectives: This study aimed to investigate the energy expenditure of common office-based tasks. The objectives were to: (a) test the classification of tasks as sedentary or light-intensity physical activity and (b) compare the energy expenditure of tasks under two postural conditions (sitting and standing).

Background: The sedentary nature of office work has been highlighted as a health risk, and strategies to reduce sedentary behavior at work have been developed. However, there is limited evidence to guide the utilization of sit-stand workstations in the workplace for metabolic health benefits.

Method: A repeated measures laboratory-based study compared the energy expenditure of common office tasks in sitting and standing using indirect calorimetry $(n=22)$. Four standardized tasks (sitting/standing quietly, reading, typing, sorting paper) under two postural conditions (sitting, standing) were performed in a randomized order.

Results: The mean energy expenditure for all tasks in sitting and standing was < I.5 METs. There were no significant differences in the energy expenditure of doing the same task in sitting compared to standing. In a repeated measures ANOVA, task $(p<.00 I)$ had a greater influence on METs expended than posture $(p=.030)$.

Conclusion: The study confirmed that the difference in energy expenditure of tasks carried out in sitting compared to standing is negligible.

Application: The ubiquitous use and utility of sitstand workstations in the workplace needs to be reviewed. Notwithstanding the potential benefits of movement that may occur naturally, this study confirmed that standing as opposed to sitting does not produce a clinically important increase in energy expenditure.

Keywords: sitting, standing, energy expenditure, occupational, sit-stand workstation

Address correspondence to Sara Dockrell, Discipline of Physiotherapy, School of Medicine, Trinity Centre for Health Sciences, St. James's Hospital, James's Street, Dublin 8, Ireland; e-mail: sara.dockrell@tcd.ie.

\section{HUMAN FACTORS}

Vol. XX, No. X, Month XXXX, pp. 1-10

DOI: $10.1177 / 0018720817719167$

Copyright (C) 2017, Human Factors and Ergonomics Society.

\section{INTRODUCTION}

Sedentary behavior, which is pervasive in contemporary society, was first highlighted as a risk factor to health in the 1950s with the landmark study on London bus workers (Morris, Heady, Raffle, Roberts, \& Parks, 1953). Once thought to be the inverse of physical activity, sedentary behavior is now considered to be a distinct paradigm that affects health independent of physical activity (Chastin, Mandrichenko, Helbostadt, \& Skelton, 2014; Hamilton, Hamilton, \& Zderic, 2007; Owen, Leslie, Salmon, \& Fotheringham, 2000) and is associated with adverse health outcomes, such as obesity, cardiovascular disease, type 2 diabetes mellitus, osteoporosis, some cancers, and all-cause mortality (Biswas et al., 2015; Grontved \& Hu, 2011; Proper, Singh, Van Mechelen, \& Chinapaw, 2011; Schmid \& Leitzmann, 2014; Van Uffelen et al., 2010; Wilmot et al., 2012).

Office workers have been identified as an occupational group that are sedentary for most of the working day (Mummery, Schofield, Steele, Eakin, \& Brown, 2005; Thorp et al. 2012), and therefore the work/office environment has been proposed as a suitable venue for public health initiatives to combat sedentary behavior (Conn, Hafdahl, Cooper, Brown, \& Lusk, 2009; McCrady \& Levine, 2009). Current public health advice to reduce total sedentary time (Garber et al., 2011) by replacing it with low-intensity non-exercise physical activity is intended to decrease the deleterious effects of work-related sedentary behavior. The beneficial effects of standing on cardio-metabolic risk biomarkers (Healy, Winkler, Owen, Anuradha, \& Dunstan, 2015) and glucose concentration (Thorp, Kingwell, Sethi, et al., 2014) have been reported, and an association between selfreported occupational sitting time and obesity in men has been found (Mummery et al., 2005). Additionally, it has been shown that using a sitstand workstation has beneficial effects on the 
musculoskeletal system (Husemann, Von Mach, Borsotto, Zepf, \& Scharnbacher, 2009; Thorp, Kingwell, Owen, \& Dunstan, 2014).

In occupational research, the effect of workplace interventions to reduce sedentariness on cognitive function or worker performance is also a focal issue. Two recent studies of call center workers concluded that standing at work increased productivity (Garrett et al., 2016) or did not result in a loss of productivity (Chau et al., 2016). In agreement with the latter study, a systematic review of workplace interventions demonstrated that there were no negative effects on self-reported work performance due to the use of sit-stand workstations (Commissaris et al., 2016). Similarly, a laboratory-based study of university employees concurred that cognitive function was not compromised when tasks were performed sitting, standing, or while walking (Ohlinger, Horn, Berg, \& Cox, 2011).

Strategies to reduce the sedentariness of office workers include the use of alternative types of office furniture, such as treadmill, pedal desks, exercise ball, and sit-stand workstations. However, the data supporting such strategies are limited (Tudor-Locke, Schuna, Frensham, \& Proenca, 2014), and the utilization of sit-stand desks provided for employees to decrease sitting time cannot be substantiated due to insufficient evidence (Shrestha et al., 2016). Although there is moderate evidence for a positive effect of sitstand workstations on sedentary behavior (Commissaris et al., 2016) and there are emerging benefits of occupational standing (Thorp, Kingwell, Owen, et al., 2014) and movement (Bailey \& Locke, 2015) compared to sitting, the current evidence is inconclusive on whether or not an increase in standing at work has a significant effect on obesity (Buckley et al., 2015). The energy expended while doing clerical work (word processing) has been reported to be significantly greater in standing compared to sitting (Beers, Roenmich, Epstein, \& Horvath, 2008), while others reported that there was no significant difference in the energy expenditure for computer work carried out in the two postures (Speck \& Schmitz, 2011). Mansoubi and colleagues (2015), who investigated the energy expenditure of different tasks and activities, also included some work-related tasks (typing) in their study but did not compare the same tasks in standing and sitting.

The definition of sedentary behavior is the cornerstone of health research in sedentary occupations and has two component parts - energy expenditure and posture (Owen, Healy, Matthews, \& Dunstan, 2010; Pate, O’Neill, \& Lobelo, 2008; Tremblay, Colley, Saunders, Healy, \& Owen, 2010). Sedentary behavior has been defined as any waking behavior characterized by an energy expenditure $\leq 1.5$ METs while in a sitting or reclining posture (Sedentary Behaviour Research Network, 2012). The MET values assigned to various activities have been developed over many years and are widely accepted (Ainsworth et al., 1993, 2011). Despite the growing body of literature on sedentary behavior, few studies to date have investigated the utility of the 1.5 METs cutoff point for sedentary behavior in the workplace. Office work typically includes a range of tasks, yet a comparison of the energy expenditure of a number of identical office-based tasks in sitting and standing has not been explored. The current study therefore aimed to test the utility of the current Sedentary Behaviour Research Network (SBRN) definition of sedentary behavior using a novel approach whereby the energy expenditure of identical tasks was measured in sitting and standing. The objectives were to (a) test the classification of the tasks as sedentary or light-intensity physical activity, namely, whether sitting tasks were $\leq 1.5$ METs (in keeping with the definition of sedentary behavior) and that the standing tasks were $>1.5$ METs, and (b) compare the energy expenditure of common office-based tasks in sitting and in standing.

\section{METHOD}

\section{Participants}

Sample size calculations were conducted using $G^{*}$ power software version 3.1.9.2 (Faul, Erdfelder, Lang, \& Buchner, 2007). An a priori power calculation revealed that with alpha $=$ 0.05 , an effect size of 0.25 , and a power of 0.95 , 22 participants were needed for this study. The effect size was calculated based on an estimated effect variance of 0.40 and an estimated error variance of 0.36 , which resulted in a medium sized partial eta squared of 0.5 (Kirk, 1996). This sample size is comparable to previous 
similar studies (Beers et al., 2008; Reiff, Marlatt, \& Dengel, 2012). Participants were recruited through postings on notice boards in the university. Participants were required to be aged between 18 and 65 years of age, provide written informed consent, and be available for testing. Those with a history of metabolic disease or any other disease affecting metabolic rate were excluded, as were those with musculoskeletal injury affecting their ability to sit or stand, cognitive impairments, chronic infectious disease, and active infections. Inclusion and exclusion criteria were chosen to reduce variation in the cohort and ensure results obtained were not affected by medical conditions. Ethical approval was granted by the Adelaide and Meath Hospital/Saint James's Hospital Joint Research Ethics Committee. Written informed consent was obtained from all participants prior to any measurements being taken, and the study was conducted in accordance with the Declaration of Helsinki.

\section{Procedure}

The study was conducted in the Exercise Laboratory of the Discipline of Physiotherapy, Trinity College Dublin. Participants were asked to abstain from caffeine and moderate to vigorous exercise for 12 hours prior to testing and fast for 4 hours prior to testing to avoid the effects of increased metabolic rate on measurements taken. When participants arrived at the laboratory, there was a familiarization period where the protocol and equipment to be used were explained to the participant and they had an opportunity to ask questions. Height and weight were measured in light clothing with shoes removed using a calibrated stadiometer (Seca model 213) and scales (Tanita HD 352), respectively.

Energy expenditure during eight tasks (four in a seated posture and the same four in a standing posture) was measured using the Cosmed $\mathrm{K} 4 \mathrm{~b}^{2}$ portable indirect calorimeter (Cosmed, Italy). Previous studies have shown the $\mathrm{K} 4 \mathrm{~b}^{2}$ to be reliable (Duffield, Dawson, Pinnington, \& Wong, 2004) and valid (Welch, Strath, \& Swartz, 2015) in the measurement of energy expenditure. Furthermore, the telemetry function of this device enabled participants to move freely at the workstation during testing as there were no cables attaching the monitor to a fixed point. As measurements took place in a laboratory, encouraging natural movement was important for this study to increase ecological validity and simulate real life. During testing, thermal comfort was achieved for all participants. The temperature was $26^{\circ} \mathrm{C}$ to $28^{\circ} \mathrm{C}$, humidity was approximately $48 \%$, and barometric pressure was 754 $\mathrm{mmHg}$. The $\mathrm{K} 4 \mathrm{~b}^{2}$ was calibrated before testing for each participant.

\section{Experimental Protocol}

To begin testing, participants were fitted with a heart rate monitor and the Cosmed $\mathrm{K} 4 \mathrm{~b}^{2}$. Participants were then asked to lie supine for 15 minutes. Resting for 15 minutes gave participants time to become comfortable wearing the Cosmed $\mathrm{K} 4 \mathrm{~b}^{2}$ before measurements of the tasks of interest were undertaken. The subsequent tasks represented common office-based activities. Each were performed in a sitting and standing posture. Task order was randomized using a random number generator. Each task was performed for 5 minutes with a 1-minute break in between tasks. A minute break was given between tasks to remind the participant which task was next, prepare any necessary equipment, and assume the correct position for the next task. The tasks were as follows: sitting quietly (no activity), sitting and reading, sitting and typing, sitting and sorting paper, standing quietly (no activity), standing and reading, standing and typing, and standing sorting paper. Tasks were carefully standardized between participants; for example, all participants read the same printed material, typed the same material, and sorted the same array of papers in the same way.

\section{Statistical Analysis}

All data were analyzed using SPSS Statistics version 22.0. MET values were calculated using the standardized formula for adults of METs = $\mathrm{VO}_{2}$ of task in $\mathrm{mL} / \mathrm{min} / \mathrm{kg}$ divided by 3.5 (Morris et al., 1993). Kilocalories expended per day were calculated using the system software of the Cosmed $\mathrm{K} 4 \mathrm{~b}^{2}$. Statistical significance was set at $p<.05$. Data were checked for normality using the Shapiro-Wilk Test. Mean and standard 
TABLE 1: Descriptive Data Presented as Mean (SD)

\begin{tabular}{lccr}
\hline Variable & Total $(n=22)$ & Female $(n=16)$ & Male $(n=6)$ \\
\hline Age, years & $27.1(7.9)$ & $27.0(8.9)$ & $27.0(5.0)$ \\
Height, cm & $170.8(6.8)$ & $168.2(5.2)$ & $177.5(6.5)$ \\
Weight, kg & $71.2(10.7)$ & $67.6(9.0)$ & $80.7(9.4)$ \\
BMl, kg/m ${ }^{-2}$ & $24.3(2.6)$ & $23.9(2.8)$ & $25.5(1.5)$ \\
\hline
\end{tabular}

deviations were calculated for baseline data and energy cost of each activity in METs. The first and last minutes of each task were discarded in the calculation of the mean energy cost for each task. Graphical analysis of means with confidence intervals were used to enable comparison between METs expended during tasks with the cutoff criteria for sedentary behavior of 1.5 METs. The difference in energy cost of the same tasks in standing compared to sitting postures was analyzed using paired $t$ tests. The overall difference between posture and tasks were analyzed using a $2 \times 4$ (posture [sitting, standing] $\times$ task [no activity, reading, typing, sorting paper]) repeated measures analysis of variance (ANOVA). In the event of a significant effect, Bonferroni tests were used for post hoc pairwise comparisons.

\section{RESULTS}

A total of 22 participants volunteered to take part in this study (16 female, 6 males). All data were normally distributed. Descriptive data for participants are detailed in Table 1. Figure 1 shows the mean and $95 \%$ CI for all tasks, including a reference line at 1.5 METs for ease of comparison with the recognized energy expenditure cutoff point for sedentary behavior. As illustrated by this graph, only one activity (standing and sorting paper) reached an energy expenditure that was not significantly lower than 1.5 METs. Table 2 details the mean $(S D)$ for each task in METs and the difference between matched tasks conducted in a seated versus a standing posture.

Paired $t$ tests revealed no significant difference between the energy cost of conducting the same task in sitting compared to standing (Table 2). To gain further information on the energy cost of the activities, a two-way repeated measures ANOVA tested whether posture (sitting or standing) or task had a significant effect on METs expended. Results revealed a significant difference between tasks, $F(3,63)=49.70$, p < $.001, n^{2}=0.703$, and posture, $F(1,21)=5.43, p$ $=.030, n^{2}=.206$, but no significant interaction between task and posture, $F(3,63)=0.782, p=$ $.509, n^{2}=0.036$. Post hoc tests revealed that overall standing resulted in a significantly greater energy expenditure than sitting, 95\% CI $[0.004,0.077]$. Sorting paper resulted in a significantly greater energy expenditure than any other task $(p<.001)$. The energy cost of typing was significantly greater than both reading and no activity (sitting/standing quietly) yet significantly less than that of sorting paper $(p<.05)$. There was no significant difference between reading and no activity.

\section{DISCUSSION}

This study explored the current definition of sedentary behavior by measuring the energy expenditure of office-based tasks in both sitting and standing. A previous study also investigated the definition of sedentary behavior using indirect calorimetry (Mansoubi et al., 2015) but included tasks performed in sitting (watching television, typing, playing PlayStation Portable and Wii) that were different from the standing tasks (standing quietly and treadmill walking at varying speeds). Two further studies compared the energy required to do a single task (computer work) while sitting and standing (Beers et al., 2008; Speck \& Schmitz, 2011). The novel approach in the current study was that a number of identical tasks (no activity, reading, typing, sorting paper) were carried out in both sitting and standing postures to allow for direct comparison.

The mean age of the participants was $27.1( \pm 7.72)$ years, which may make the findings most applicable to younger adults rather than 


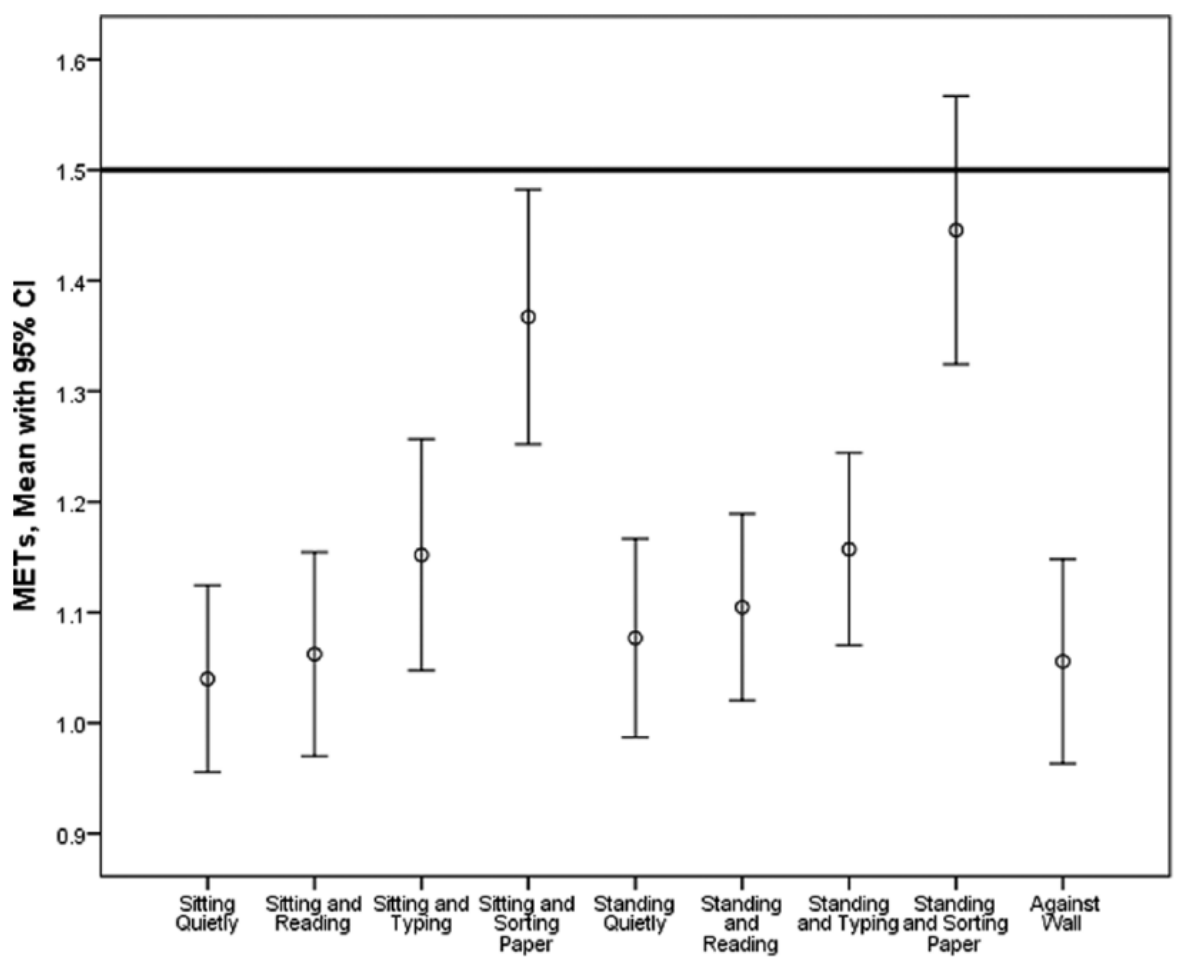

Figure 1. Mean and 95\% CI for each task compared with 1.5 METs.

across the life span. However, this is a worthwhile demographic to have sampled as during their 20 s and 30 s, people are typically starting office-based working careers, working long hours, and forming habitual office behaviors. These habitual office-based behaviors could be carried on for the rest of their working lives.

A main finding of this study was that the mean energy expenditures for all tasks carried out in sitting were below 1.5 METs. All the sitting tasks therefore complied with the classification and definition of sedentary behavior. This is in agreement with others (Newton, Han, Zderic, \& Hamilton, 2013; Speck \& Schmitz, 2011) who also found that sitting-based behaviors (watching television, reading, typing) had a MET value of less than 1.5 METs. Mansoubi et al. (2015) reported that all of the seated tasks they examined, except playing the Wii, were on average less than 1.5 METs. Playing the Wii while seated expended on average 2.06 METs. In keeping with their findings, it was expected in the current study that the task of sorting paper would exceed the cutoff point for sedentary behavior, but in reality, the mean MET value of the task in sitting was 1.37. The benefits of accumulating non-exercise activity, especially among sedentary populations, have been raised (Hamilton et al., 2007; Levine, Schleusner, \& Jensen, 2000), and therefore the identification of tasks and activities that contribute to this is of importance and warrants further investigation.

An unexpected finding was that the mean energy expenditure during all of the standing tasks was less than 1.5 METs. The average MET value of 1.3 attributed to standing quietly in the Compendium of Physical Activities (Ainsworth et al., 2011) is based on 21 studies. Five of these used indirect calorimetry to measure the energy cost of standing in a population comparable to the current study (Crouter, Clowers, \& Bassett, 2006; Kanade, Gokhale, \& Rao, 2001; Kuriyan, Easwaran, \& Kurpad, 2006; Lanningham-Foster et al., 2009; Levine et al., 2000). Of the five studies, all reported the energy cost of standing quietly to be below 1.5 METs, and all except one (Kanade et al., 2001) found the energy cost to be below 1.3 METs. A recent study on the energy 
TABLE 2: MET Values for Each Task in Seated and Standing Posture and Difference Between Postures

\begin{tabular}{lccc}
\hline Task & $\begin{array}{c}\text { Seated } \\
\text { Mean }(S D)\end{array}$ & $\begin{array}{c}\text { Standing } \\
\text { Mean }(S D)\end{array}$ & $\begin{array}{c}\text { Difference } \\
\mathrm{p}[95 \% \mathrm{CI}]\end{array}$ \\
\hline No activity & $1.04(0.19)$ & $1.08(0.20)$ & $0.274[-0.105 .0 .314]$ \\
Reading & $1.06(0.21)$ & $1.10(0.19)$ & $0.148[-0.102,0.016]$ \\
Typing & $1.15(0.24)$ & $1.16(0.20)$ & $0.863[-0.068,0.057]$ \\
Sorting paper & $1.37(0.26)$ & $1.45(0.27)$ & $0.085[-0.169,0.012]$ \\
\hline
\end{tabular}

cost of sitting and standing also reported low MET values for standing: $0.90 \pm 0.18$ for men and $0.87 \pm 0.11$ for the women (Júdice, Hamilton, Sardinha, Zderic, \& Silva, 2016). Not all studies to date consistently report standing quietly to be less than 1.5 METs. Mansoubi et al. (2015) found that standing quietly/still expended 1.6 METs. Nonetheless, from the findings of our study and the limited number of studies using calorimetry to quantify the energy cost of standing, it can be deduced that the average energy cost of standing quietly is likely to be lower than the 1.5 MET cutoff point for sedentary behavior. Furthermore, it appears that there is a large range of mean values varying from 0.9 to 1.6 METs. Given the similarities of the participants across the studies and the use of objective, valid, and reliable measures to quantify the energy cost of sitting and standing, the relatively broad range may be explained by the individual variability that is known to exist (Levine et al., 2005).

The only task in the current study that was not statistically significantly lower than the 1.5 METs cutoff point was standing and sorting paper. There is no known study with which direct comparison of this finding could be made, but Levine et al. (2000) allocated a value of 1.8 METs to the activity of standing, fidgeting. A key issue that arises is that the majority of officebased standing tasks satisfied the energy component of the definition of sedentary behavior, with energy expenditure being less than 1.5 METs, the threshold value that distinguishes sedentary behavior from light-intensity physical activity. Although this leads us to question whether officebased activities at a sit-stand workstation should be considered a sedentary behavior, the findings should also be interpreted in the context of the real world. Similar to others (Júdice et al., 2016; Mansoubi et al., 2015), the current study was carried out in a laboratory. Using portable equip- ment, accurate measurements of simulated work activities were ensured in the laboratory setting, but the worker behavior associated with the use of a sit-stand workstation in an office may be different. An office-based study using activity monitors observed that stepping time as well as standing was significantly greater in the intervention group (adjustable sit-stand workstation) than the comparison group (nonadjustable sitting workstation) after one week (Alkhajah et al., 2012). However, the positive behavioral response to the novel office equipment was not maintained for stepping time at the 3-month follow up.

The current MET value assigned to standing, reading in the 2011 Compendium of Physical Activities is not based on objective data but is estimated at 1.8 METs (Ainsworth et al., 2011). However, the findings of this study would suggest that the energy cost of the activity has been overestimated. The Compendium is a live document, and therefore the objective high-quality data reported in this paper can contribute to the expected MET values cited for standing, reading and other seated and standing tasks in the next published update of the Compendium.

The findings of this study suggest that the difference in energy expenditure between identical sitting and standing tasks can be negligible. There was no significant difference between the energy costs of doing the same task in sitting compared to standing. This is in agreement with Speck and Schmitz (2011), who also found no significant difference for the measured computer activity while sitting and standing. In contrast, Beers et al. (2008) reported a significant difference; however, they did not provide the MET values. Additionally, Beers et al. presented the results graphically, so direct comparisons could not be made. Healy et al. (2015) noted that the potential benefits from breaking up sedentary time are dependent on the activities that replace 
the sedentary time. They concluded that substituting standing for sitting was effective in reducing some risks of cardiovascular disease but was not sufficient to affect indicators of adiposity. In keeping with Healy et al., the evidence from the current study would support the finding that simply replacing sitting with standing does not have a clinically significant effect on energy expenditure and therefore adiposity/body weight.

The manner in which sedentary behavior is accumulated is important. Periods of prolonged sedentary behavior are detrimental to health (Buckley et al., 2015; Healy et al., 2008; Robertson, Ciriello, \& Garabet, 2013; Swartz, Squires, $\&$ Strath, 2011), and breaking up sedentary time is the accepted public health advice for the prevention of work-related ill health. There has been an increased demand for sit-stand desks in recent years, in accordance with the advice and growing interest in changing sedentary office working environments (Buckley et al., 2015; Robertson et al., 2013; Swartz et al., 2011). However, the optimum utility of sit-stand workstations has yet to be explored. Beers et al. (2008) concluded that standing had the advantage over sitting for promoting physical activity; however, the participants in their laboratory-based study reported that standing was less comfortable and more tiring than sitting on an office chair. A recent Cochrane review examined the value of workplace interventions in the reduction of workrelated sitting time (Shrestha et al., 2016). The review included an evaluation of the use of sitstand workstations and concluded that the quality of evidence that the use of sit-stand desks reduces workplace sitting time was very low.

It has been demonstrated in the current study that the difference in energy expenditure was minimal when tasks were performed sitting compared to standing. It has been suggested that meaningful increases in energy expenditure could be accrued through the actual transfer from sitting to standing and vice versa (Júdice et al., 2016). Therefore, the transition from one posture to another may be the all important component. That is, standing is of no particular benefit, but the change in posture has the greatest effect on energy expenditure. Therefore, more frequent breaks or changes in posture should be recommended rather than simply recommending periods of standing over sitting.
Alkhajah et al. (2012) noted that the number of sit-stand transitions was greater in those at a sitstand workstation compared to those at a seated workstation, even though postural change was not a variable under investigation. Good standing posture is favorable as it causes minimal stress or strain on the body and provides an optimum position for the function of the thoracic and abdominal organs (Galley \& Forster, 1987). Although considered to be an optimum posture, prolonged standing at work is not advocated due to the potential of acquiring musculoskeletal problems (Andersen, Haahr, \& Frost, 2007). Walking while working is also favorable and can be performed without compromising cognitive function (Ohlinger et al., 2011). Guidelines have been developed to promote the avoidance of sustained periods of sedentary behavior in the workplace (Buckley et al., 2015), and a system-based approach to sedentary behavior has been proposed (Chastin et al., 2016). These guidelines and proposals are welcomed editions to educating the workforce on the deleterious effects of sedentary behavior and also highlighting the direction of further research in this area to determine what causes the health benefits associated with breaking up sedentary time and provide evidence-based guidance for the optimum use of sit-stand workstations.

This study found that the task conducted had a greater effect on energy expended than the posture it was conducted in. This finding would suggest that to increase the energy cost of office-based activities, they should be more dynamic rather than simply being conducted in a different posture. In terms of increasing energy expenditure, a series of upper limb or lower limb movements could have a beneficial effect (Levine et al., 2000). Alternatively, sedentary time should be broken up with periods of more dynamic activity. Since tasks in the current study were chosen to represent typical office activities and were not considered to be particularly demanding, it is surprising that they had a greater effect on overall energy cost than posture.

\section{Strengths and Limitations}

The use of portable indirect calorimetry equipment to measure energy expenditure, which was the main dependent variable, was a 
primary strength of this study. Accurate values of energy expenditure were recorded with limited interference to the participants while they conducted the various tasks. In this way, direct comparisons could be made for the independent variables of interest. Additionally, the findings of this study could provide novel data for the next Compendium of Physical Activities.

MET values for tasks were calculated using the system software of the indirect calorimeter (by dividing $\mathrm{VO}_{2}$ by 3.5) and were not calculated from individual's resting metabolic rate (RMR). It was not an objective of the current study to measure RMR; furthermore, to adhere to the gold standard methods of measuring RMR, a different indirect calorimeter that is compatible with a hood would have been needed. The tasks measured in the current study could not be performed using a hood, therefore two methods of data collection would have been needed to calculate one value, which is not ideal. Although it has been argued that the value of 3.5 $\mathrm{ml} \mathrm{O} \cdot \mathrm{kg}^{-1} \cdot \mathrm{min}^{-1}$ is an overestimation of 1 MET (Byrne, Hills, Hunter, Weinsier, \& Schutz, 2005), the method used here to calculate METs is widely accepted and has been used in similar studies (Júdice et al., 2016; Mansoubi et al., 2015).

Given the limited range of BMI within the sample participants, the effects of BMI could not be explored, and the sample is not representative of the general population. A further limitation of the study was that movement or stepping that can occur in the real-world setting were not included as test conditions. However, the overall aim of the study was to explore energy expenditure of sitting and standing at a workstation, as it has previously been established that moving around would increase energy expenditure (Swartz et al., 2011).

\section{CONCLUSION}

The current study explored the SBRN definition of sedentary behavior using indirect calorimetry to measure energy expenditure. The study showed that occupational sitting tasks had a MET value of less than 1.5 METs. Standing tasks were also less than 1.5 METs, that is, below the current energy expenditure threshold for classification as sedentary behavior. The task that was undertaken had a greater influence on the energy expenditure than the posture of the person. This study adds to the body of knowledge of how to define sedentary behavior and presented a novel comparison of occupational tasks while sitting and standing. Furthermore, the study presented objective data for an activity (standing, reading) that had previously been assigned an estimated value in the Compendium of Physical Activities. The study also confirms that standing as opposed to sitting does not produce a clinically important increase in energy expenditure in a laboratory setting over short periods of time. Further research to clarify the definition and understanding of sedentary behavior in the workplace is warranted to better inform future workplace sedentary behavior guidelines and preventive strategies.

\section{ACKNOWLEDGMENTS}

We thank the participants who gave so freely of their time.

\section{KEY POINTS}

- The mean energy expenditure for office tasks while sitting and standing was $<1.5$ METs.

- The energy cost of individual tasks sitting versus standing were not significantly different.

- Task undertaken had a greater influence on energy expenditure than posture.

- Substituting standing for sitting is not adequate to produce metabolic health benefits.

- Novel objective values for tasks are presented here and can contribute to the next Compendium of Physical Activities.

\section{REFERENCES}

Ainsworth, B. E., Haskell, W. L., Herrmann, S. D., Meckes, N., Bassett, D. R., Jr., Tudor-Locke, C., . . . Leon, A. S. (2011). 2011 compendium of physical activities: A second update of codes and MET values. Medicine and Science in Sports and Exercise, 43, 1575-1581.

Ainsworth, B. E., Haskell, W. L., Leon, A. S., Jacobs, D. R., Jr., Montoye, H. J., Sallis, J. F., \& Paffenbarger, R. S., Jr. (1993). Compendium of physical activities: Classification of energy costs of human physical activities. Medicine and Science in Sports and Exercise, 25, 71-80.

Alkajah, T., Reeves, M., Eakin, E., Winkler, E., Owen, N., \& Healy, G. (2012). Sit-stand workstations: A pilot intervention to reduce office sitting time. American Journal of Preventive Medicine, 43, 298-303. 
Andersen, J. H., Haahr, J. P., \& Frost, P. (2007). Risk factors for more severe regional musculoskeletal symptoms: A two-year prospective study of a general working population. Arthritis and Rheumatism, 56, 1355-1364.

Bailey, D., \& Locke, C. (2015). Breaking up prolonged sitting with light-intensity walking improves postprandial glycemia, but breaking up sitting with standing does not. Journal of Science and Medicine in Sport, 18, 294-298.

Beers, E., Roenmich, J., Epstein, L., \& Horvath, P. (2008). Increasing passive energy expenditure during clerical work. European Journal of Applied Physiology, 103, 353-360.

Biswas, A., Oh, P. I., Faulkner, G. E., Bajaj, R. R., Silver, M. A., Mitchell, M. S., \& Alter, D. A. (2015). Sedentary time and its association with risk for disease incidence, mortality, and hospitalization in adults: A systematic review and meta-analysis. Annals of Internal Medicine, 162, 123-132.

Buckley, J. P., Hedge, A., Yates, T., Copeland, R. J., Loosemore, M., Hamer, M., . . . Dunstan, D. W. (2015). The sedentary office: An expert statement on the growing case for change towards better health and productivity. British Journal of Sports Medicine, 49, 1357-1362.

Byrne, N. M., Hills, A. P., Hunter, G. R., Weinsier, R. L., \& Schutz, Y. (2005). Metabolic equivalent: One size does not fit all. Journal of Applied Physiology, 99, 1112-1119.

Chastin, S. F., De Craemer, M., Lien, N., Bernaards, C., Buck, C., Oppert, J-M., . . Cardon, G. (2016). The SOS-framework (systems of sedentary behaviours): An international transdisciplinary consensus framework for the study of determinants, research priorities and policy on sedentary behaviour across the life course: A DEDIPAC study. International Journal of Behavioral Nutrition and Physical Activity, 13, 83. doi:10.1186/s12966-016-0409-3

Chastin, S. F., Mandrichenko, O., Helbostadt, J. L., \& Skelton, D. A. (2014). Associations between objectively-measured sedentary behaviour and physical activity with bone mineral density in adults and older adults, the NHANES study. Bone, 64, 254-262.

Chau, J., Sukula, W., Fedel, K., Do, A., Engelen, L., Kingham, M., .. Bauman, A. (2016). More standing and just as productive: Effects of a sit-stand desk intervention on call center workers' sitting, standing, and productivity at work in the Opt to Stand pilot study. Preventive Medicine Reports, 3, 68-74.

Commissaris, D., Huysmans, M., Mathiassen, S., Srinivasan, D., Koppes, L., \& Hendriksen, I. (2016). Interventions to reduce sedentary behaviour and increase physical activity during productive work: A systematic review. Scandinavian Journal of Work, Environment and Health, 42, 181-191.

Conn, V. S., Hafdahl, A. R., Cooper, P. S., Brown, L. M., \& Lusk, S. L. (2009). Meta-analysis of workplace physical activity interventions. American Journal of Preventive Medicine, 37, 330-339.

Crouter, S. E., Clowers, K. G., \& Bassett, D. R., Jr. (2006). A novel method for using accelerometer data to predict energy expenditure. Journal of Applied Physiology, 100, 1324-1331.

Duffield, R., Dawson, B., Pinnington, H. C., \& Wong, P. (2004). Accuracy and reliability of a Cosmed K4b2 portable gas analysis system. Journal of Science and Medicine in Sport, 7, 11-22.

Faul, F., Erdfelder, E., Lang, A. G., \& Buchner, A. (2007). G*Power 3: A flexible statistical power analysis program for the social, behavioral, and biomedical sciences. Behavior Research Methods, 39, 175-191.

Galley, P., \& Forster, A. (1987). Human movement-An introductory text for physiotherapy students. Edinburgh: Churchill Livingstone.
Garber, C. E., Blissmer, B., Deschenes, M. J., Franklin, B. A., Lamonte, M. J., Lee, I. M., ... Swain, D. P. (2011). American College of Sports Medicine position stand. Quantity and quality of exercise for developing and maintaining cardiorespiratory, musculoskeletal, and neuromotor fitness in apparently healthy adults: Guidance for prescribing exercise. Medicine and Science in Sports and Exercise, 43, 1334-1359.

Garrett, G., Benden, M., Mehta, R., Pickens, A., Peres, S. C., \& Zhao, H. (2016). Call center productivity over 6 months following a standing desk intervention. IIE Transactions on Occupational Ergonomics and Human Factors, 4, 2-3. doi: 10.1080/21577323.2016.1183534

Grontved, A., \& Hu, F. B. (2011). Television viewing and risk of type 2 diabetes, cardiovascular disease, and all-cause mortality: A meta-analysis. Journal of the American Medical Association, 305, 2448-2455.

Hamilton, M. T., Hamilton, D. G., \& Zderic, T. W. (2007). Role of low energy expenditure and sitting in obesity, metabolic syndrome, type 2 diabetes, and cardiovascular disease. Diabetes, $56,2655-2667$.

Healy, G. N., Dunstan, D. W., Salmon, J., Cerin, E., Shaw, J. E., Zimmet, P. Z., \& Owen, N. (2008). Breaks in sedentary time: Beneficial associations with metabolic risk. Diabetes Care, 31, 661-666.

Healy, G. N., Winkler, E. A., Owen, N., Anuradha, S., \& Dunstan, D. W. (2015). Replacing sitting time with standing or stepping: Associations with cardio-metabolic risk biomarkers. European Heart Journal, 36, 2643-2649.

Husemann, B., Von Mach, C., Borsotto, D., Zepf, K., \& Scharnbacher, J. (2009). Comparisons of musculoskeletal complaints and data entry between a sitting and a sit-stand workstation paradigm. Human Factors, 51, 310-320.

Júdice, P. B., Hamilton, M. T., Sardinha, L. B., Zderic, T. W., \& Silva, A. M. (2016). What is the metabolic and energy cost of sitting, standing and sit/stand transitions? European Journal of Applied Physiology, 116, 263-273.

Kanade, A. N., Gokhale, M. K., \& Rao, S. (2001). Energy costs of standard activities among Indian adults. European Journal of Clinical Nutrition, 55, 708-713.

Kirk, R. E. (1996). Practical significance: A concept whose time has come. Educational and Psychological Measurement, 56, 746-759.

Kuriyan, R., Easwaran, P. P., \& Kurpad, A. V. (2006). Physical activity ratio of selected activities in Indian male and female subjects and its relationship with body mass index. British Journal of Nutrition, 96, 71-79.

Lanningham-Foster, L., Foster, R. C., McCrady, S. K., Jensen, T. B., Mitre, N., \& Levine, J. A. (2009). Activity-promoting video games and increased energy expenditure. Journal of Pediatrics, 154, 819-823.

Levine, J. A., Lanningham-Foster, L. M., McCrady, S. K., Krizan, A. C., Olson, L. R., Kane, P. H., . . Clark, M. M. (2005). Interindividual variation in posture allocation: Possible role in human obesity. Science, 307, 584-586.

Levine, J. A., Schleusner, S. J., \& Jensen, M. D. (2000). Energy expenditure of nonexercise activity. American Journal of Clinical Nutrition, 72, 1451-1454.

Mansoubi, M., Pearson, N., Clemes, S. A., Biddle, S. J., Bodicoat, D. H., Tolfrey, K., . . . Yates, T. (2015). Energy expenditure during common sitting and standing tasks: Examining the 1.5 MET definition of sedentary behaviour. BMC Public Health, 15, 516. doi:10.1186/s12889-015-1851-x

McCrady, S. K., \& Levine, J. A. (2009). Sedentariness at work: How much do we really sit? Obesity, 17, 2103-2105. 
Morris, C. K., Myers, J., Froelicher, V. F., Kawaguchi, T., Ueshima, K., \& Hideg, A. (1993). Nomogram based on metabolic equivalents and age for assessing aerobic exercise capacity in men. Journal of the American College of Cardiology, 22, 175-182.

Morris, J. N., Heady, J. A., Raffle, P. A., Roberts, C. G., \& Parks, J. W. (1953). Coronary heart-disease and physical activity of work. Lancet, 265, 1111-1120.

Mummery, W. K., Schofield, G. M., Steele, R., Eakin, E. G., \& Brown, W. J. (2005). Occupational sitting time and overweight and obesity in Australian workers. American Journal of Preventive Medicine, 29, 91-97.

Newton, R. L., Jr., Han, H., Zderic, T., \& Hamilton, M. T. (2013). The energy expenditure of sedentary behavior: A whole room calorimeter study. PLoS One, 8, e63171.

Ohlinger, C., Horn, T., Berg, W., \& Cox, R. (2011). The effect of active workstation use on measures of cognition, attention, and motor skill. Journal of Physical Activity and Health, 8, 119-125.

Owen, N., Healy, G. N., Matthews, C. E., \& Dunstan, D. W. (2010). Too much sitting: The population health science of sedentary behavior. Exercise and Sports Science Reviews, 38, 105-113.

Owen, N., Leslie, E., Salmon, J., \& Fotheringham, M. J. (2000). Environmental determinants of physical activity and sedentary behavior. Exercise and Sports Science Reviews, 28, 153-158.

Pate, R. R., O’Neill, J. R., \& Lobelo, F. (2008). The evolving definition of "sedentary." Exercise and Sports Science Reviews, 36, 173-178.

Proper, K. I., Singh, A. S., Van Mechelen, W., \& Chinapaw, M. J. (2011). Sedentary behaviors and health outcomes among adults: A systematic review of prospective studies. American Journal of Preventive Medicine, 40, 174-182.

Reiff, C., Marlatt, K., \& Dengel, D. (2012). Difference in caloric expenditure in sitting versus standing desks. Journal of Physical Activity and Health, 9, 1009-1011.

Robertson, M. M., Ciriello, V. M., \& Garabet, A. M. (2013). Office ergonomics training and a sit-stand workstation: Effects on musculoskeletal and visual symptoms and performance of office workers. Applied Ergonomics, 44, 73-85.

Schmid, D., \& Leitzmann, M. F. (2014). Television viewing and time spent sedentary in relation to cancer risk: A meta-analysis. Journal of the National Cancer Institute, 106(7). doi:10.1093/ jnci/dju098

Sedentary Behaviour Research Network. (2012). Letter to the editor: Standardized use of the terms "sedentary" and "sedentary behaviours." Applied Physiology Nutrition and Metabolism, 37, 540-542.

Shrestha, N., Kukkonen-Harjula, K. T., Verbeek, J. H., Ijaz, S., Hermans, V., \& Bhaumik, S. (2016). Workplace interventions for reducing sitting at work. Cochrane Database Systematic Reviews, 3, CD010912.

Speck, R., \& Schmitz, K. (2011). Energy expenditure comparison: A pilot study of standing instead of sitting at work for obesity prevention. Preventive Medicine, 52, 283-284.

Swartz, A. M., Squires, L., \& Strath, S. J. (2011). Energy expenditure of interruptions to sedentary behavior. International Journal of Behavioral Nutrition and Physical Activity, 8, 69.

Thorp, A. A., Healy, G. N., Winkler, E., Clark, B. K., Gardiner, P. A., Owen, N., \& Dunstan, D. W. (2012). Prolonged sedentary time and physical activity in workplace and non-work contexts: A cross-sectional study of office, customer service and call centre employees. International Journal of Behavioral Nutrition and Physical Activity, 9, 128.

Thorp, A. A., Kingwell, B. A., Owen, N., \& Dunstan, D. W. (2014). Breaking up workplace sitting time with intermittent standing bouts improves fatigue and musculoskeletal discomfort in overweight/obese office workers. Occupational and Environmental Medicine, 71, 765-771.

Thorp, A. A., Kingwell, B. A., Sethi, P., Hammond, L., Owen, N., \& Dunstan, D. W. (2014). Alternating bouts of sitting and standing attenuate postprandial glucose responses. Medicine and Science in Sports and Exercise, 46, 2053-2061.

Tremblay, M. S., Colley, R. C., Saunders, T. J., Healy, G. N., \& Owen, N. (2010). Physiological and health implications of a sedentary lifestyle. Applied Physiology Nutrition and Metabolism, 35, 725-740.

Tudor-Locke, C., Schuna, J., Frensham, L., \& Proenca, M. (2014). Changing the way we work: Elevating energy expenditure with workstation alternatives. International Journal of Obesity, 38 , 755-765.

Van Uffelen, J. G., Wong, J., Chau, J. Y., Van Der Ploeg, H. P., Riphagen, I., Gilson, N. D., . . Brown, W. J. (2010). Occupational sitting and health risks: A systematic review. American Journal of Preventive Medicine, 39, 379-388.

Welch, W. A., Strath, S. J., \& Swartz, A. M. (2015). Congruent validity and reliability of two metabolic systems to measure resting metabolic rate. International Journal of Sports Medicine, 36, 414-418.

Wilmot, E. G., Edwardson, C. L., Achana, F. A., Davies, M. J., Gorely, T., Gray, L. J., . . B Biddle, S. J. (2012). Sedentary time in adults and the association with diabetes, cardiovascular disease and death: Systematic review and meta-analysis. Diabetologia, 55, 2895-2905.

Jill Burns is a chartered physiotherapist. Since graduating, she worked on a Marie Curie FP7 project investigating furniture in schools, prior to undertaking her MSc research. Jill is currently working in a private physiotherapy practice in Dublin.

Cuisle Forde is an assistant professor in the Discipline of Physiotherapy. Her research is in the area of exercise and physical activity levels in pediatric and clinical populations, including those with chronic communicable diseases.

Sara Dockrell is an assistant professor in the Discipline of Physiotherapy, School of Medicine, Trinity College Dublin. Her primary research interest is the investigation and application of ergonomics to promote physical health and well-being in educational and workplace settings.

Date received: October 21, 2016

Date accepted: June 4, 2017 